\title{
Postoperative symptoms of psychosis after deep brain stimulation in patients with Parkinson's disease
}

\author{
Azam A. Qureshi, BA, ${ }^{1}$ Jennifer J. Cheng, MD, MS, ${ }^{2}$ Abraham N. Sunshine, BS, ${ }^{2}$ \\ Adela Wu, ScB/AB, ${ }^{3}$ Gregory M. Pontone, MD, ${ }^{4}$ Nicola Cascella, MD, ${ }^{5}$ Frederick A. Lenz, MD, PhD, ${ }^{2}$ \\ Stephen E. Grill, MD, PhD, ${ }^{6}$ and William S. Anderson, PhD, MD ${ }^{2}$

\begin{abstract}
1 University of Maryland School of Medicine; '2Department of Neurosurgery, Johns Hopkins Hospital; ${ }^{3}$ Johns Hopkins School of Medicine; ${ }^{4}$ Department of Psychiatry, Johns Hopkins School of Medicine; ${ }^{5}$ Sheppard and Enoch Pratt Hospital, Baltimore; and ${ }^{6}$ The Parkinson's and Movement Disorders Center of Maryland, Elkridge, Maryland
\end{abstract}

\begin{abstract}
OBJECT Cases of postoperative psychosis in Parkinson's disease patients receiving deep brain stimulation (DBS) treatment have previously been published. However, the magnitude of symptom incidence and the clinical risk factors are currently unknown. This retrospective study sheds light on these issues by investigating psychosis in a group of 128 Parkinson's disease patients who received DBS implants.
\end{abstract}

METHODS A retrospective chart review was performed to obtain surgery dates, follow-up clinic visit dates, and associated stimulation parameter settings (contacts in use and the polarity of each along with stimulation voltage, frequency, and pulse width) for each patient. Unified Parkinson's Disease Rating Scale II Thought Disorder scores, used as a clinical assessment tool to evaluate the presence of psychosis at each visit, were also collected. The data were compiled into a database and analyzed.

RESULTS The lifetime incidence of psychosis in this cohort of patients was $28.1 \%$. The data suggest that risk of psychosis remains fairly constant throughout the first 5 years after implantation of a DBS system and that patients older at the time of receiving the first DBS implant are not only more likely to develop psychosis, but also to develop symptoms sooner than their younger counterparts. Further analysis provides evidence that psychosis is largely independent of the clinically used electrode contact and of stimulation parameters prior to psychosis onset.

CONCLUSIONS Although symptoms of psychosis are widely seen in patients with Parkinson's disease in the years following stimulator placement, results of the present suggest that most psychoses occurring postoperatively are likely independent of implantation and stimulation settings.

http://thejns.org/doi/abs/10.3171/2015.3.FOCUS1523

KEY WORDS deep brain stimulation; Parkinson's disease; psychosis; neurosurgery; psychiatry

$\mathrm{D}$ EEP brain stimulation (DBS) surgery, a procedure widely used in Parkinson's disease (PD) treatment, involves the implantation of a quadripolar electrode, an extension cable, and an implantable pulse generator, ${ }^{17}$ which electrically alter the pathophysiological activity of specific regions of the brain. ${ }^{17}$ When applied to the subthalamic nucleus (STN-DBS) or globus pallidus interna (GPi-DBS), this therapy has been shown to alleviate the characteristic PD motor symptoms of tremor, bradykinesia, and rigidity. ${ }^{2}$ The STN is often preferred because of its compact target size,${ }^{16}$ and DBS is often applied bilat- erally. ${ }^{14}$ The optimal stimulation location for therapeutic effect is individually determined for each case based on patient evaluation, preoperative MR images or CT scans, intraoperative microelectrode recording, and, often, intraoperative stimulation. ${ }^{2}$ The mechanisms of action for the therapeutic effects of DBS, however, are complex and not entirely clear. ${ }^{2}$ Postoperatively, the polarity, voltage, pulse width, and frequency of stimulation at the DBS electrode contacts are adjusted over time to maximize therapeutic benefit and the battery life of the neurostimulator, while minimizing undesirable side effects.

ABBREVIATIONS DBS = deep brain stimulation; GPi-DBS = globus pallidus interna-DBS; PD = Parkinson's disease; STN-DBS = subthalamic nucleus-DBS; UPDRS = Unified PD Rating Scale; UPDRS II TD = UPDRS II Thought Disorder.

SUBMITTED January 16, 2015. ACCEPTED March 16, 2015.

INCLUDE WHEN CITING DOI: 10.3171/2015.3.FOCUS1523.

DISCLOSURE Dr. Grill reports being a consultant for Medtronic. 
There are several reported nonmotor side effects that can result from DBS therapy in PD patients (targeting either the STN or the GPi) including varying degrees of cognitive changes and changes in mood, ${ }^{14}$ symptoms of mania or hypomania, ${ }^{15}$ depression, ${ }^{15}$ and even suicide. ${ }^{3}$ Postoperative symptoms of psychosis have also been reported in PD patients receiving DBS treatment. ${ }^{9,11,18,21} \mathrm{Al}-$ though potential mechanisms of DBS-induced psychosis have been hypothesized, including the postoperative lesion effect and the vulnerability of limbic circuitry in the $\mathrm{STN},{ }^{18}$ symptom incidence rate and clinical risk factors remain unknown.

Psychosis, characterized by the presence of hallucinations and delusions, has been studied in the general PD population, ${ }^{6}$ with published prevalence rates ranging from $17 \%$ to $72 \% .^{6}$ The wide variation in reported prevalence may be in part due to methodological differences in the use of various scales and questionnaires, such as the Unified Parkinson's Disease Rating Scale (UPDRS) or the Neuropsychiatric Inventory, to screen for specific symptoms of psychosis. ${ }^{6}$ Despite these discrepancies, even the lower range of published values suggests that psychosis affects many PD patients. Although both PD itself ${ }^{6}$ and antiparkinsonian medications $s^{6,8}$ are thought to contribute to symptoms, the relationship between psychosis in PD patients and DBS treatment has not yet been explored.

This retrospective study examined psychosis in a group of 173 PD patients, most of whom received DBS implants at Johns Hopkins Hospital between 1998 and 2011. To characterize the potential risk for patients of developing psychosis, a survival curve and incidence rates for psychosis were quantified and are presented here. Psychiatric evaluations, as determined by UPDRS II Thought Disorder (UPDRS II TD) scores, were also analyzed with respect to the time since the first DBS implant, age, PD duration, sex, stimulation parameters, and active contact depth.

\section{Methods \\ Patients}

A retrospective chart review was performed utilizing medical records from a large tertiary care movement disorder practice. This retrospective study was approved by the Johns Hopkins Medicine Institutional Review Board. One hundred seventy-three patients who had received either unilateral or bilateral STN-DBS (97.1\%) or GPi-DBS (1.7\%) implants for idiopathic PD treatment between 1998 and 2011 were identified. An additional 1.2\% of patients received both STN-DBS and GPi-DBS. These patients presented to a movement disorder practice that involved 3 separate neurosurgeons (in other words, we chose a time interval, and these were the subjects seen at that practice who were referred for and actually underwent DBS surgery). The movement disorder practice is a multidisciplinary practice in Maryland that manages patients with Parkinson's disease medically; it has in-house neuropsychology and physical therapy. This practice made the referral for DBS surgery, and it followed the subjects postoperatively for DBS optimization and further medical management. Electronic patient records at Johns Hopkins
Hospital were also searched to determine any missing date, stimulation, or hospital information. The patient population was $25.4 \%$ female and $74.6 \%$ male, and the patients were evaluated by a multidisciplinary team consisting of neurologists, neuropsychologists, and neurosurgeons to assess their candidacy for the procedure. Approximately $53 \%$ of these patients had undergone surgery at Johns Hopkins Hospital, while the remaining patients received implants at various other institutions. Data recorded from patient charts included the specific stimulation settings and the UPDRS II TD scores, both at the clinic visit immediately prior to DBS implantation and at subsequent visits following the surgery.

\section{Unified Parkinson's Disease Rating Scale II Thought Disorder Score}

The UPDRS II TD score was used as the primary clinical indicator of psychosis in this study. This score is derived from the UPDRS assessment tool, which collectively evaluates the motor function and independence in activities of daily living of PD patients. ${ }^{5}$ The UPDRS II TD score, which specifically monitors hallucinations and delusions, ranges from 0 to 4 , with 0 corresponding to an absence of symptoms, 1 corresponding to intense dreaming, 2 corresponding to hallucinations with retained insight, 3 corresponding to more common hallucinations or delusions lacking insight and possibly interfering with daily activities, and 4 corresponding to persistent symptoms that render the patient unable to care for him or herself. ${ }^{10}$ UPDRS II TD scores were recorded for each patient at routine follow-up and reprogramming visits to the movement disorder clinic following stimulator placement. Additionally, each patient's UPDRS II TD score from the clinic visit immediately prior to the first DBS implantation was used to describe a baseline psychological state.

UPDRS II TD scale-derived psychosis criteria were previously described in a study of the general PD patient population, in which psychosis was defined as a score of 2 or greater. ' In the current study, psychosis was considered to have occurred in those patients in whom a preoperative UPDRS II TD score of 0 or 1 increased by at least 2 points.

The data suggest that, in many cases, psychotic symptoms were transient and, in fewer cases, the symptoms were more long-lived. However, due to the methodological limitations of using data from clinic visits occurring at highly variable rates from patient to patient, it is difficult to reliably quantify the duration of each case of psychotic symptoms. For many patients, UPDRS TD II scores indicated intermittent states of psychoses as defined in this study. For the purposes of this study, an incidence of psychosis was considered as the first instance of a qualifying UPDRS II TD score, regardless of the progression of scores at subsequent visits.

\section{Statistical Analysis}

In addition to UPDRS II TD scores, we also retrieved surgery dates, clinic visit dates, and stimulation parameter settings (contacts in use and the polarity of each along with stimulation voltage, frequency, and pulse width) from 
records for each patient at follow-up and reprogramming visits. The data were compiled into a database and analyzed using Microsoft Excel and SAS Statistical Software.

Patients were excluded from all analyses if they had missing preoperative UPDRS II TD scores or scores that were 2 or greater (totaling 40 patients, as shown in Table 1). Additionally, patients were excluded if they were missing $>30 \%$ of reported follow-up UPDRS II TD scores prior to psychosis incidence for those who developed symptoms or, for patients without incident psychosis, missing > $30 \%$ of scores prior to the last clinic visit (6 total). These groups of patients were excluded due to the inability to characterize psychosis incidence without a baseline score, unreliable reporting of psychosis onset time, or unreliable estimate of psychosis incidence.

To examine the possible effects of stimulation characteristics on the development of psychosis, 2 separate comparisons were made between patients who developed psychosis $(n=35)$ and a control group of 35 selected patients who did not develop psychosis but matched the psychosis group in terms of sex composition, mean age at first device implantation, and mean age at visit of interest (either the incidence of psychosis or random visit). There were 26 males and 9 females in the control group, which had mean ages at first implantation and at the selected visit of 61.4 years and 65.4 years, respectively. There were 26 males and 9 females in the group with incident psychosis, which had mean ages at first implantation and at the visit of incidence of 63.1 years and 66.0 years, respectively.

Further protocol for each analysis in addition to handling time reference points and incomplete data are detailed in supplemental material (see Appendix for details).

All mean values are reported \pm the SD unless otherwise noted. Proportions were compared using Fisher's exact test, while continuous variables were compared using unpaired t-tests for unequal variances utilizing the software previously described. All $\mathrm{p}$ values reported are 2 tailed.

\section{Results}

\section{Incident Psychosis}

From a total of 173 patients, 128 met the criteria to be included for calculation of incident psychosis. Using the previously described criteria, the incidence of psychosis for patients with baseline UPDRS II TD scores of 0 or 1 during the study period was found to be $28.1 \%$ (36/128) or 5.8 cases per 100 person-years from time of first implantation. There was an average of $5.0 \pm 2.7$ years of follow-up after the first DBS device placement in our patient sample.

TABLE 1. Preoperative UPDRS II TD scores

\begin{tabular}{cc}
\hline Score & No. of Patients \\
\hline 0 & 113 \\
\hline 1 & 20 \\
\hline 2 & 8 \\
\hline 3 & 1 \\
NR & 31 \\
\hline Total no. of patients & 173 \\
\hline
\end{tabular}

$\mathrm{NR}=$ not reported.
For those patients whose baseline UPDRS II TD scores were 0 prior to surgery, the incidence of psychosis was found to be $29.4 \%(32 / 109)$ or 6.4 cases per 100 personyears. A smaller incidence rate $(21.1 \%$ [4/19] or 3.4 cases per 100 person-years) was found for patients with preoperative UPDRS II TD scores of 1 , but this difference was not statistically significant.

Year to year incidence rates for 1-5 years after first implantation were calculated for the 128 patients with baseline UPDRS II TD scores of 0 or 1 (excluding 3 patients with vague first implantation dates) (Table 2). No significant differences in rates during this period were found, which suggests that the risk of psychosis remains fairly constant throughout the first 5 years after implantation. Cumulative incidence rates for 1 to 5 years after first implant were also calculated (Table 3).

Kaplan-Meier survival analysis of the same 125 patients from the 1 to 5-year incidence rate sample was also carried out with respect to the development of psychosis. Survival probability, or the proportion of patients remaining without incident psychosis at a given year after the first implantation, dropped from approximately 0.93 at 1 year postimplantion to approximately 0.76 at 4 years postimplantion (Fig. 1).

\section{Age, PD Duration, and Sex}

Older age is thought to be associated with hallucinations in PD patients. ${ }^{1,6,13}$ Ages at the time of first implantation were compared between patients who developed psychosis and those who did not develop psychosis. Results showed that patients who eventually developed psychosis during the study period $(n=35)$ were significantly older at the time of their first implantation $(\mathrm{p}<0.007)$ than patients who did not develop symptoms $(\mathrm{n}=90)$ (Fig. 2).

These results were underscored by the finding that older patients at the time of first implantation were at progressively higher risk for developing postoperative symptoms of psychosis. Specifically, psychosis developed in $10.0 \%$ of those aged 34-49 years at first surgery, $25.0 \%$ of those aged 49-64 years, and $40.0 \%$ of those aged 64-79 years (Fig. 3, Table 4). The difference in proportion of psychosis incidences between the oldest and youngest groups was significant $(\mathrm{p}<0.02)$.

Because evidence indicated advanced age as a potential risk factor for psychosis onset in these patients, time from first implantation to psychosis incidence was also compared among the same age groups. The results suggest that those patients who were older at the time of the

\section{TABLE 2. Year to year incidence of psychosis*}

\begin{tabular}{ccc}
\hline No. of Patients & Yrs After Implant & Incidence Rate (\%) \\
\hline 114 & $0-1$ & 5.3 \\
\hline 109 & $1-2$ & 8.3 \\
\hline 97 & $2-3$ & 5.2 \\
\hline 86 & $3-4$ & 4.7 \\
\hline 53 & $4-5$ & 5.7 \\
\hline
\end{tabular}

* Incidence rates for Years 1-5 after first implantation in patients with a preoperative UPDRS II TD score of 0 or 1 . No single difference in rates was statistically significant. 
TABLE 3. Cumulative incidence of postoperative psychosis*

\begin{tabular}{ccc}
\hline No. of Patients & Yrs After Implant & Incidence Rate (\%) \\
\hline 114 & 1 & 5.3 \\
\hline 109 & 2 & 13.8 \\
\hline 97 & 3 & 18.6 \\
\hline 86 & 4 & 20.9 \\
\hline 53 & 5 & 32.1 \\
\hline
\end{tabular}

* Cumulative incidence rates for Years 1-5 after first implantation in patients with a preoperative UPDRS II TD score of 0 or 1.

first implantation who developed psychosis had symptoms manifest sooner after the first surgery than their younger counterparts. Notably, significant differences in years from first implantation to psychosis incidence existed between patients who were aged 49-64 years $(\mathrm{n}=15,3.7 \pm$ 2.7 years) and $64-79$ years $(\mathrm{n}=18,1.9 \pm 1.7$ years $)$ at the time of first implantation ( $<$ 0.04). (Fig. 3, Table 4).

Mean patient age was also compared between both groups of patients at the time of the last follow-up visits. The comparison showed a significantly older mean age at the last follow-up visits in patients who developed psychosis $(69.1 \pm 7.8$ years) than in patients who did not develop symptoms $(62.6 \pm 9.2$ years $)(p<0.0002)$ (Table 5). Therefore, both groups were not followed to comparably advanced ages. Mean age at time of psychosis incidence (in patients who developed psychosis) was compared with the mean age at the last recorded clinic visit (in patients who did not develop psychosis) to examine the extent of this age difference by focusing on age at psychosis onset. Despite a discrepancy, no significantly older age at psychosis incidence was found (Table 5).

Longer duration of disease is also thought to be associated with psychosis in PD. ${ }^{6,20}$ Mean PD duration at time of the last follow-up visits was compared for both groups of patients to explore the possibility that more patients might have developed symptoms if they were followed up further into the disease course. Results showed that, on average, patients developing psychosis were followed up significantly longer into the disease course $(17.5 \pm 5.2$ years) than patients without symptoms $(14.4 \pm 5.8$ years) $(\mathrm{p}$

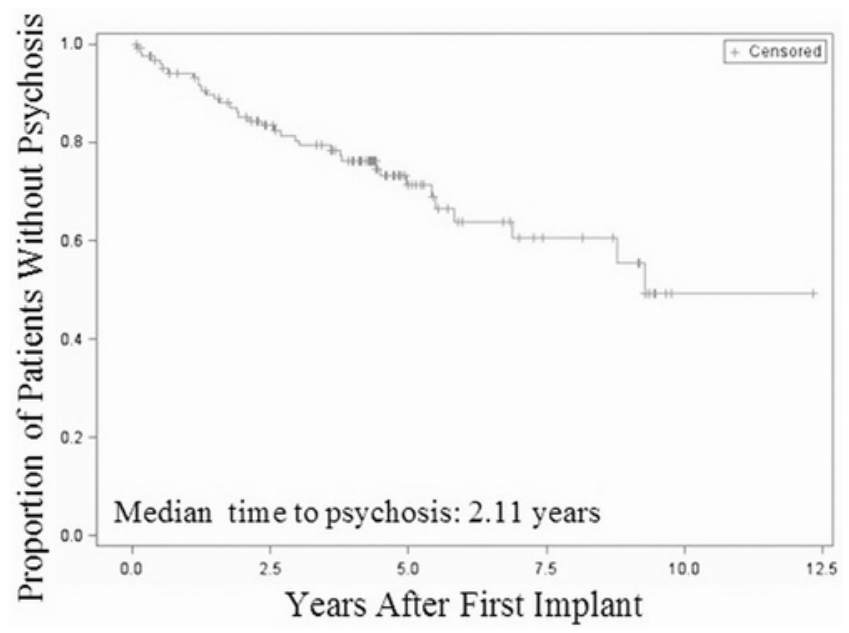

FIG. 1. Kaplan-Meier survival curve for psychosis incidence.

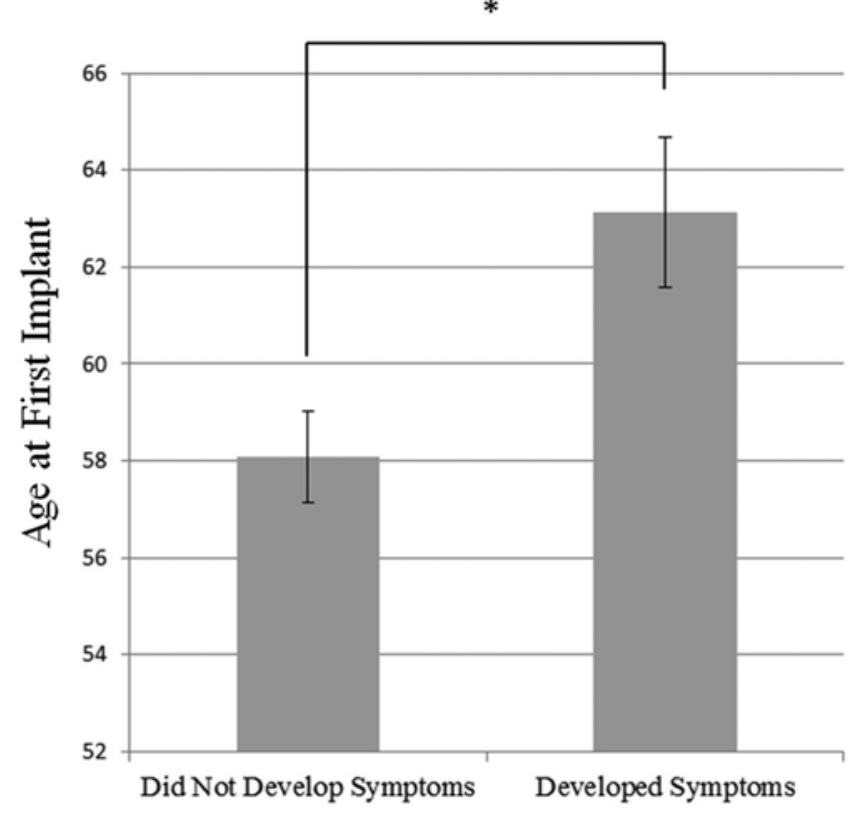

FIG. 2. Age discrepancy at time of first implantation of a DBS system. Patients who did not develop psychosis during the study period $(n=90)$ received their first implants at a mean age of $58.1 \pm 8.9$ years, whereas patients who eventually developed psychosis $(n=35)$ received their first implants at a mean age of $63.1 \pm 9.2$ years $\left({ }^{*} p<0.007\right)$. Error bars indicate standard error of the mean, and the 2-tailed $p$ value was determined using the 2-sample t-test for unequal variances.

$<0.005$ ) (Table 5). Additionally, no significant difference was found in a comparison between the mean PD duration at symptom onset in patients developing symptoms (14.4 \pm 4.9 years) and the mean PD duration at the last visit in patients not developing symptoms $(14.4 \pm 5.8$ years) (Table 5). Compared to the mean PD duration at symptom onset, the absence of a longer mean PD duration at last visit in patients without symptoms suggests that these patients may not have been followed up sufficiently long enough into the disease course to develop symptoms, and this supports our earlier age results. Together, these results suggest that patients lacking symptoms were not followed as long, either in age or into the disease course, as those who developed symptoms. Because of the discrepancy in age at time of first implantation between patients who developed psychosis and those who did not, the mean PD duration at time of first implantation was compared between these groups of patients as well. No significant differences were found (Table 5). This result suggests that older age may be a stronger risk factor than disease duration for psychosis after DBS implantation in PD patients.

Patient sex was also compared between the group of patients developing psychosis and the group of patients lacking symptoms: The former group was composed of $80.6 \%$ males (29/36), while the latter group was composed of $75.0 \%$ males (69/92) (data not shown). This difference was not significant and suggests that males and females are equally susceptible to psychosis incidence after implantation of a DBS device. As there is a higher incidence of PD in men than in women, the composition of the sample population was consistent with that observed across the general PD population. ${ }^{19}$ 


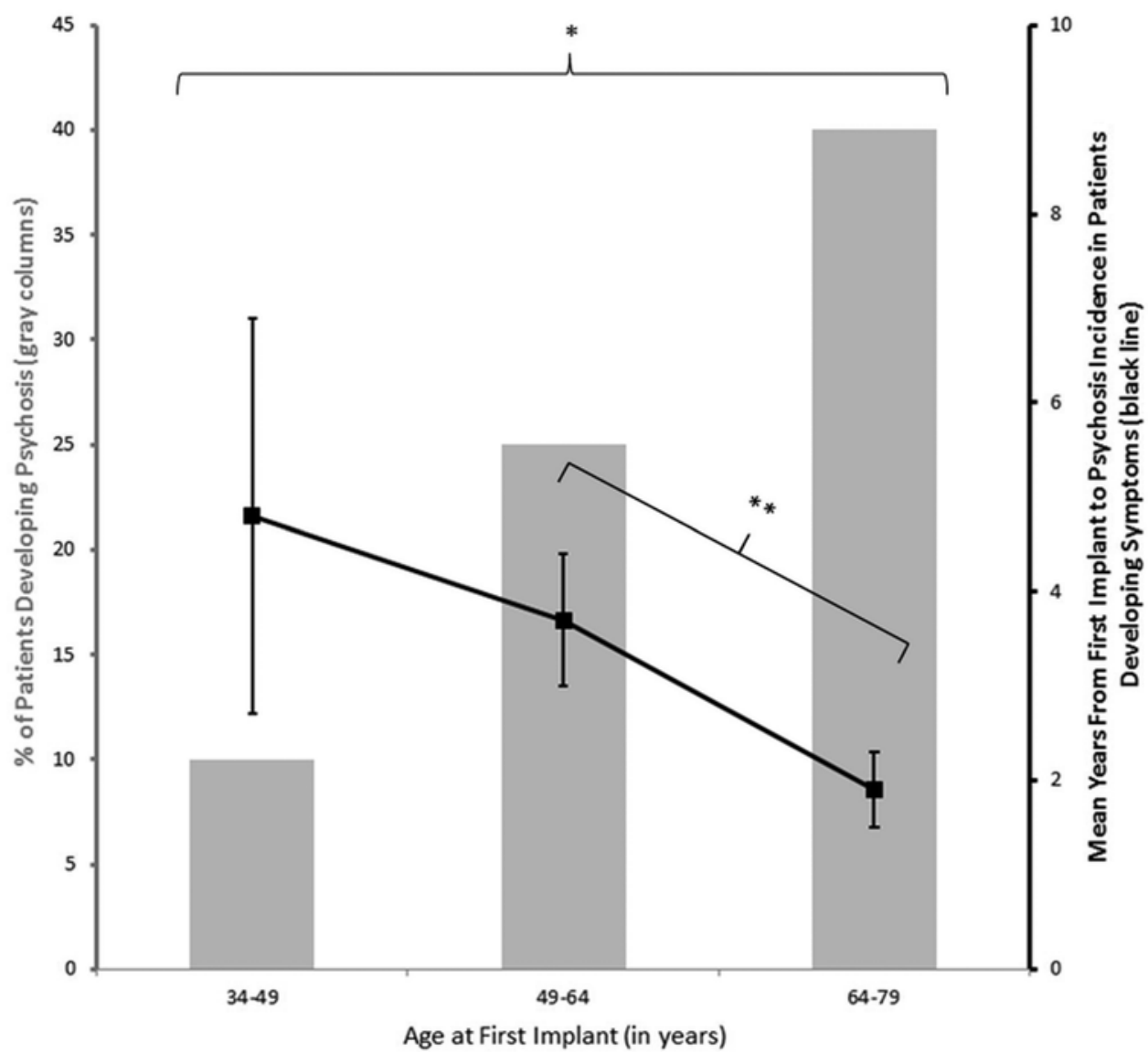

FIG. 3. Age at time of first implantation and time to incidence. The gray columns show that $10.0 \%$ of patients $34-49$ years old at the time of receiving the first DBS implant $(n=20)$ developed psychosis, while $25.0 \%$ and $40.0 \%$ of those in the age groups ranging from 49 to 64 years $(n=60)$ and $64-79$ years $(n=45)$, respectively, developed psychosis. The incidence difference between the youngest and oldest age group was statistically significant ( ${ }^{*} p<0.02$, 2-tailed $p$ value obtained from Fisher's exact test). The black line shows that patients 34-49 years old at the time of receiving the first DBS implant who developed psychosis $(n=2)$ did so a mean of $4.8 \pm 3.0$ years after their first surgeries, while those in age groups ranging from 49 to 64 years $(n=15)$ and 64 to 79 years $(n=18)$ developed psychosis a mean of $3.7 \pm 2.7$ and $1.9 \pm 1.7$ years, respectively, after first implantation. Error bars indicate standard error of the mean. The difference between the two oldest groups was statistically significant $\left({ }^{* *} p<0.04,2\right.$-tailed $p$ value obtained from 2-sample t-test for unequal variances). Table 4 summarizes the results.

\section{Stimulation Parameters and Contact Depth}

DBS parameters, including frequency, voltage, and pulse width (or duration), were analyzed with respect to psychosis incidence using methods previously described. No significant differences in any of these parameters were found in comparisons between the matched control group and the group of affected patients (Tables 6 and 7).

Active stimulation of the deepest electrode contact has been previously implicated in causing reversible adverse neuropsychiatric effects. ${ }^{4}$ Proportions of deepest contacts

\section{TABLE 4. Analysis of psychosis development and age at first} implantation

\begin{tabular}{cccc}
\hline $\begin{array}{c}\text { Age at 1st } \\
\text { Implant (yrs) }\end{array}$ & $\begin{array}{c}\text { No. of } \\
\text { Patients }\end{array}$ & $\begin{array}{c}\text { \% Developing } \\
\text { Psychosis }\end{array}$ & $\begin{array}{c}\text { Yrs From 1st Implant to } \\
\text { Psychosis Incidence* }\end{array}$ \\
\hline $34-49$ & 20 & 10.0 & $4.8 \pm 3.0(n=2)$ \\
\hline $49-64$ & 60 & 25.0 & $3.7 \pm 2.7(n=15)$ \\
\hline $64-79$ & 45 & 40.0 & $1.9 \pm 1.7(n=18)$ \\
\hline
\end{tabular}

* Values are expressed as mean \pm SD in patients developing symptoms. actively being stimulated at the visit prior to the development of psychosis were also compared among these groups and also yielded no significant results (Fig. 4).

\section{Discussion}

This study found that patients receiving their first stimulator implant at an older age tended to develop symptoms more frequently than those receiving their first implant at younger ages. This alone suggests either that older patients at time of first implantation are more susceptible to psychosis or that there is an association, independent of the implant, between age and psychosis. The findings that older patients experienced symptoms sooner than younger patients suggests a relationship between older age and psychosis symptom onset similar to what has been reported previously. ${ }^{1,6,13}$ Interestingly, patients with incident psychosis were followed up longer on average than those lacking symptoms.

The analysis of PD duration suggests that patients with incident psychosis and patients without incident psychosis were not followed up equally long into the disease course, 
TABLE 5. Summary of results from mean age and mean PD duration comparisons

\begin{tabular}{|c|c|c|c|}
\hline \multirow[b]{2}{*}{ Factor } & \multicolumn{2}{|c|}{$\begin{array}{l}\text { Age of Patients or PD Duration } \\
\text { in Yrs }\end{array}$} & \multirow[b]{2}{*}{$\mathrm{p}$ Value } \\
\hline & $\begin{array}{l}\text { w/ Psychosis } \\
\quad(n=35)\end{array}$ & $\begin{array}{l}\text { w/o Psychosis } \\
\quad(n=90)\end{array}$ & \\
\hline \multicolumn{4}{|l|}{ Mean age } \\
\hline At first implant & 63.1 & 58.1 & $<0.007$ \\
\hline $\begin{array}{l}\text { At psychosis incidence } \\
\text { or last visit }\end{array}$ & $66.0^{*}$ & $62.6 \dagger$ & NS \\
\hline At last visit & 69.1 & 62.6 & $<0.0002$ \\
\hline \multicolumn{4}{|l|}{ Mean PD duration } \\
\hline At first implant & 11.5 & 9.8 & NS \\
\hline $\begin{array}{l}\text { At psychosis incidence } \\
\text { or last visit }\end{array}$ & $14.4^{*}$ & $14.4 \dagger$ & NS \\
\hline At last visit & 17.5 & 14.4 & $<0.005$ \\
\hline
\end{tabular}

just as they were not followed up to equally advanced ages. Therefore, it is possible that patients without psychosis might have developed psychosis had they been followed for longer, and the possibility exists that PD duration might play a key role in psychosis after DBS. According to a study by Yoritaka et al. of a cohort of 1453 PD patients, longer PD duration appears to correspond with increasing incidences of psychosis. ${ }^{20}$ In fact, $33.8 \%$ of all PD patients in their cohort had developed psychosis by 12 years. ${ }^{20}$ Psychosis was only present in $15.4 \%$ of patients followed up for at least 12 years after PD onset in the present study (data not shown). This comparatively lower incidence is encouraging, although Yoritaka et al. did not specify if any of their patients received DBS therapy. Still, a definitive conclusion associating PD duration and psychosis cannot be drawn from the data presented in the present study.

A 12-year population-based study of psychosis in PD patients conducted by Forsaa et al. used similar methodology to ours and found a lifetime psychosis incidence rate of $41.9 \%$ in a total sample of 160 patients who lacked such symptoms in their history. Forsaa et al. only considered

TABLE 6. Mean point parameter comparisons between patients developing psychosis and controls*

\begin{tabular}{lcc}
\hline & \multicolumn{2}{c}{ Mean Value (no. of patients) } \\
\cline { 2 - 3 } Mean Parameters & $\begin{array}{c}\text { Patients Developing } \\
\text { Psychosis Prior to Incidence }\end{array}$ & $\begin{array}{c}\text { Control Group Prior } \\
\text { to Selected Visit }\end{array}$ \\
\hline Rt frequency $(\mathrm{Hz})$ & $177.5(26)$ & $185.0(30)$ \\
\hline Rt voltage $(\mathrm{V})$ & $4.2(25)$ & $2.9(30)$ \\
\hline Rt duration $(\mu \mathrm{sec})$ & $99.2(26)$ & $112.0(30)$ \\
\hline Lt frequency $(\mathrm{Hz})$ & $181.5(30)$ & $185.0(32)$ \\
\hline Lt voltage $(\mathrm{V})$ & $2.7(30)$ & $3.1(32)$ \\
\hline Lt duration $(\mu \mathrm{sec})$ & $108.7(30)$ & $100.3(32)$ \\
\hline
\end{tabular}

* Means of point parameters in the visits immediately prior to psychosis incidence in patients developing changes and prior to selected visits in the matched control group were compared. No significant differences were found.
TABLE 7. Comparison between average of the 300-day weighted means in patients who developed psychosis and controls*

\begin{tabular}{|c|c|c|}
\hline \multirow[b]{2}{*}{ Mean Parameters } & \multicolumn{2}{|c|}{$\begin{array}{l}\text { Average of the Weighted Mean Value } \\
\text { (no. of patients) } \dagger\end{array}$} \\
\hline & $\begin{array}{l}\text { Patients Developing } \\
\text { Psychosis Over } 300 \text {-Day } \\
\text { Course Prior to Incidence }\end{array}$ & $\begin{array}{l}\text { Control Group Over } \\
\text { 300-Day Course Prior } \\
\text { to Selected Visit }\end{array}$ \\
\hline Rt frequency $(\mathrm{Hz})$ & $181.9(17)$ & $184.0(30)$ \\
\hline Rt voltage (V) & $2.7(17)$ & $2.9(30)$ \\
\hline Rt duration ( $\mu \mathrm{sec})$ & $91.1(17)$ & $109.2(30)$ \\
\hline Lt frequency $(\mathrm{Hz})$ & $182.2(23)$ & $184.8(32)$ \\
\hline Lt voltage $(\mathrm{V})$ & $2.7(23)$ & $3.1(32)$ \\
\hline Lt duration ( $\mu \mathrm{sec}$ ) & $107.2(23)$ & $99.2(32)$ \\
\hline
\end{tabular}

\footnotetext{
* Mean weighted means for parameters in the 300 days leading up to incidence were compared to those in the 300 days leading up to the selected visits in the control group. No significant differences were found.

$\dagger$ Average of the weighted means indicates the following: for each patient a weighted means was computed, and then the average value for each parameter was determined.
}

patients with a baseline UPDRS II TD score of 0; the same subset of patients in the current study was found to have an incident psychosis rate of $29.6 \%$. The discrepancy could be due to differing follow-up times or older patient ages, as mean follow-up time after the initiation of the Forsaa et al. study was not presented, and the patients in their study had a mean older age ( 75.7 years and 72.6 years at baseline for those who developed and those who did not develop psychosis, respectively) than the patients in this study (63.1 years and 58.1 years, respectively). Regardless, the incidence of psychosis in this study was less than the published incidence of psychosis in the general PD population as reported by Forsaa et al., and although they did not state whether any patients received DBS implants, this finding is promising in that it does not indicate any increased risk of psychosis for PD patients who underwent DBS implantation, a concern sparked by case reports of psychosis developing postoperatively. ${ }^{9,11,18,21}$

Of particular interest to this study was the group of 8 patients who developed symptoms within 1 year after DBS system implantation, a timeframe similar to that reported in previous case studies (Table 8)., ${ }^{911,18,21}$ These patients represent $22.2 \%$ (8 of 36) of all patients who developed symptoms in the present study. Nonetheless, the incidence of psychosis in the 1st year after implantation was similar to that found in the 2nd, 3rd, 4th, and 5th years following placement of the DBS device. Taken together with evidence concerning patient age, PD duration, stimulation parameter, and active contact depth analysis, findings from this study suggest that DBS implantation and stimulation settings do not seem to be primarily responsible for the majority of psychoses occurring postoperatively.

\section{Study Limitations}

There are several limitations of this study. First, there are two main drawbacks to gauging PD onset by first diagnosis date or date of first symptom onset, which were the only available indicators in the present study: This methodology introduces an aspect of unreliability because di- 


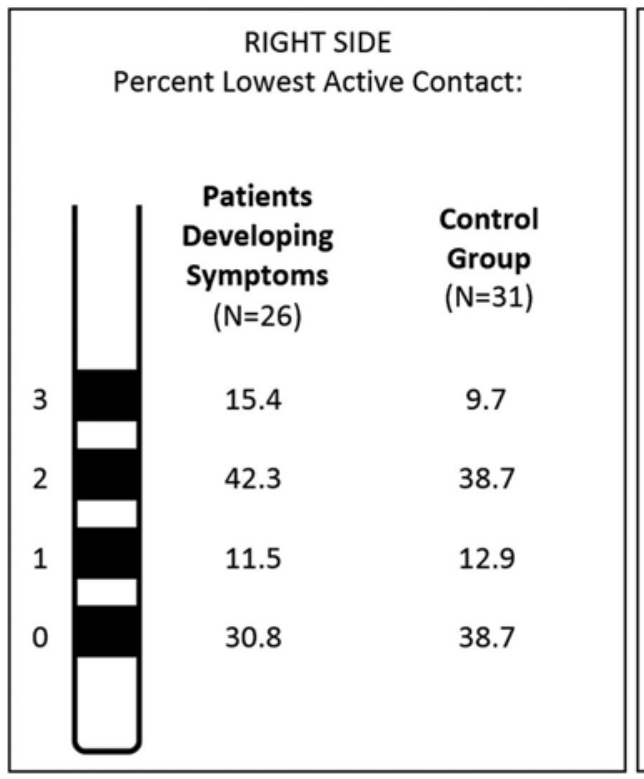

\begin{tabular}{|c|cc|}
\hline \multicolumn{3}{|c|}{ LEFT SIDE } \\
Percent Lowest Active Contact: \\
\\
\end{tabular}

FIG. 4. Analysis of deepest active stimulation contacts. The depths of all 4 potential stimulation contacts on each electrode are represented. Proportions of deepest active stimulation contacts in patients developing symptoms were compared with those of patients in the control group. No significant differences were found.

agnosis dates likely occurred at varying times after actual disease onset, and detection of initial symptoms by the patient can be subjective. ${ }^{12}$ Another limitation is that we did not evaluate the effects of antiparkinsonian medications on psychosis onset, as these medications have been shown to influence psychosis in PD. 6,8 Dopaminergic drugs used to treat motor symptoms of PD are associated with induction of psychosis, possibly through mediating imbalances in multiple neurotransmitter systems in the brain. ${ }^{8}$ The main objective of this study, however, was primarily to examine incident psychosis after DBS in PD patients with respect to DBS implantation. Lastly, the UPDRS II TD scale used to monitor psychosis in this group of patients has been previously criticized for inadequately evaluating clinical areas of mentation, mood, and behavior. ${ }^{10}$ Despite this, it is a commonly used metric for evaluating psychosis in $\mathrm{PD}^{6}$ and, moreover, the score is a readily attainable metric that was routinely recorded at follow-up visits. Despite these limitations, this study has characterized the risk of psychosis developing up to 5 years after first DBS implantation, which was the mean follow-up period for patients in this study.

\section{Future Directions}

Future work should seek to make further comparisons

TABLE 8. Case studies reporting psychosis after DBS

\begin{tabular}{lcc}
\hline Case Study & $\begin{array}{c}\text { Time to Psychosis } \\
\text { Incidence After Implant }\end{array}$ & $\begin{array}{c}\text { Psychosis Incidence } \\
\text { Prior to Stimulation }\end{array}$ \\
\hline Herzog et al., 2003 & $1 \mathrm{mo}$ & No; 3 wks after \\
\hline $\begin{array}{l}\text { Raucher-Chéné et al., } \\
2008\end{array}$ & $1 \mathrm{yr}$ & Not mentioned \\
\hline Zonana et al., 2011 & 3 days & Yes \\
\hline Widge et al., 2013 & $1-2$ wks & Yes \\
\hline
\end{tabular}

between incidence rates of psychosis in the non-DBS PD population and the DBS PD population, while implementing standardized methodology for psychosis screening and controlling for follow-up time, anti-PD medications, disease course, and age of PD patients. In doing so, the objective of determining the differences between psychosis developing in PD as a result of non-DBS factors will become more evident and more distinct from the factors influencing rare, abrupt psychosis described in case studies, which have attributed it to DBS treatment. ${ }^{9,11}$ As these cases of psychosis continue to develop in PD patients receiving DBS, there is need for a better understanding of the risk and etiology of these adverse symptoms.

\section{Conclusions}

This study quantified the incidence of psychosis in PD patients after DBS implantation. Specifically, a lifetime incidence rate of $28.3 \%$ was found, with psychosis being defined as an increase of 2 or more points from a preoperative UPDRS II TD score of 0 or 1 . Additional findings from this study provide evidence for the following: 1) the risk of psychosis remains fairly constant throughout the first 5 years after DBS device implantation, 2) patients older at the time of the first implantation are at a higher risk for developing psychosis, 3) patients receiving implants at older ages who develop psychosis do so sooner after the first implantation than those receiving implants at younger ages, 4) men and women are equally likely to develop symptoms, and 5) active stimulation contact depth and stimulation parameters in cases in which psychosis develops are not primary influences on the development of psychosis in the majority of cases.

Taken together, evidence suggests that a majority of psychoses occurring postoperatively are not directly due to DBS device implantation. This study also corroborates previous evidence linking hallucinations, the primary 
component of psychosis as defined in this study, in PD patients to advanced age., ${ }^{1,6,13}$ The breadth of novel findings in this study should be useful to clinicians assessing patient candidacy for the DBS procedure commonly used in the treatment of PD and to patients giving informed consent for the surgery.

\section{Appendix \\ Methods}

The time variable considered in this study was in relation to the patient's first implant. The first implant was used as a starting point for evaluation because, although many patients underwent bilateral DBS implantation in the same surgery (74/128 patients meeting inclusion criteria), others received bilateral implants in staged surgeries (33/128) or received unilateral implants (17/128). There were an additional 4 patients whose records were unclear as to whether the bilateral implants were placed in the same surgery or staged surgeries. Patients whose first implantation dates were unclear from patient records $(9 / 128)$ were only included in analyses involving surgery dates if the actual date was narrowed down to a time span of less than 1 year (6/9). For these 6 patients with small windows of time that included the actual surgery date, the first date in the range was used as the working surgery date.

For PD duration analysis, the earliest indication of PD from the charts (either first symptoms date or diagnosis date) was considered as the starting point of PD for each patient. If only a month and/or year was reported and a distinct date was not found in the charts, the 1st day of the reported month and/or year was used as the working PD start date. For 5 patients, the indicated year of first symptoms was recorded. For 107 patients, the indicated year of diagnosis was recorded. For 10 patients, the month and year of diagnosis were both recorded. Specific dates of diagnoses were recorded for 4 patients, and the first possible day was used as the working PD start date for the 2 remaining patients who had 1) a 2-year span of possible dates and 2) a latest possible limit for the diagnosis date.

Total lifetime incidence rates for the development of psychosis were determined for 3 sets of patients. The first 2 sets were those patients with preoperative UPDRS II TD scores of 0 and 1 , respectively, while the third set was a combination of the previous 2 sets. All further analyses were carried out using only the third and largest set of patients. For cumulative psychosis incidence rates over 1-5 years, only patients for whom data had been recorded for the length of time being analyzed were considered. For noncumulative year to year incidence rates, only the patients followed up throughout the entire year were considered, along with any who developed psychosis during that year but did not attend follow up to the completion of that year.

For comparisons of parameter points, the mean parameters from the visits immediately prior to psychosis incidence in the group of patients developing symptoms were compared to the mean parameters from the visits immediately prior to selected visits in the control group. The selected visits, 1 for each member in the control group, were only chosen if there were 300 days of fully reported data prior to the visits because the same visits were used for the 300-day weighted mean parameter comparison. For the 300-day weighted mean parameter comparison, the weighted means for each parameter in patients who developed symptoms were computed for a 300-day range immediately prior to psychosis incidence. A mean of these figures was compared to that from the weighted means accounting for the 300 days leading up to visits in the control group.

For the comparison of contact depths, the proportion of deepest contacts in use at the time of the visit immediately prior to psychosis incidence was compared to the proportion of deepest contacts in use prior to the control group of visits.

\section{Acknowledgments}

We are grateful to Drs. Frederick Lenz, Zach Levine, and Howard Eisenberg for performing the surgeries on this cohort of patients.

\section{References}

1. Aarsland D, Larsen JP, Cummins JL, Laake K: Prevalence and clinical correlates of psychotic symptoms in Parkinson disease: a community-based study. Arch Neurol 56:595-601, 1999

2. Anderson WS, Lenz FA: Surgery insight: Deep brain stimulation for movement disorders. Nat Clin Pract Neurol 2:310-320, 2006

3. Appleby BS, Duggan PS, Regenberg A, Rabins PV: Psychiatric and neuropsychiatric adverse events associated with deep brain stimulation: A meta-analysis of ten years' experience. Mov Disord 22:1722-1728, 2007

4. Bejjani BP, Damier P, Arnulf I, Thivard L, Bonnet AM, Dormont D, et al: Transient acute depression induced by highfrequency deep-brain stimulation. N Engl J Med 340:14761480, 1999

5. Chou KL, Taylor JL, Patil PG: The MDS-UPDRS tracks motor and non-motor improvement due to subthalamic nucleus deep brain stimulation in Parkinson disease. Parkinsonism Relat Disord 19:966-969, 2013

6. Fénelon G, Alves G: Epidemiology of psychosis in Parkinson's disease. J Neurol Sci 289:12-17, 2010

7. Forsaa EB, Larsen JP, Wentzel-Larsen T, Goetz CG, Stebbins GT, Aarsland D, et al: A 12-year population-based study of psychosis in Parkinson disease. Arch Neurol 67:996-1001, 2010

8. Friedman JH: Parkinson's disease psychosis 2010: a review article. Parkinsonism Relat Disord 16:553-560, 2010

9. Herzog J, Reiff J, Krack P, Witt K, Schrader B, Müller D, et al: Manic episode with psychotic symptoms induced by subthalamic nucleus stimulation in a patient with Parkinson's disease. Mov Disord 18:1382-1384, 2003

10. Holroyd S, Currie LJ, Wooten GF: Validity, sensitivity and specificity of the mentation, behavior and mood subscale of the UPDRS. Neurol Res 30:493-496, 2008

11. Raucher-Chéné D, Charrel CL, de Maindreville AD, Limosin F: Manic episode with psychotic symptoms in a patient with Parkinson's disease treated by subthalamic nucleus stimulation: improvement on switching the target. J Neurol Sci 273:116-117, 2008

12. Reider CR, Halter CA, Castelluccio PF, Oakes D, Nichols WC, Foroud T: Reliability of reported age at onset for Parkinson's disease. Mov Disord 18:275-279, 2003

13. Sanchez-Ramos JR, Ortoll R, Paulson GW: Visual hallucinations associated with Parkinson disease. Arch Neurol 53:1265-1268, 1996

14. Smith Y, Wichmann T, Factor SA, DeLong MR: Parkinson's disease therapeutics: new developments and challenges since the introduction of levodopa. Neuropsychopharmacology 37:213-246, 2012

15. Temel Y, Kessels A, Tan S, Topdag A, Boon P, Visser-Vandewalle V: Behavioural changes after bilateral subthalamic stimulation in advanced Parkinson disease: a systematic review. Parkinsonism Relat Disord 12:265-272, 2006

16. Vives KP: Neuromodulation procedures for movement disorders, in Ghogawala Z, Krishnaney AA, Steinmetz MP, et al (eds): The Evidence for Neurosurgery. Shrewsbury: tfm Publishing Limited, 2012, pp 155-164

17. Volkmann J, Herzog J, Kopper F, Deuschl G: Introduction to the programming of deep brain stimulators. Mov Disord 17 (Suppl 3):S181-S187, 2002

18. Widge AS, Agarwal P, Giroux M, Farris S, Kimmel RJ, Hebb 
AO: Psychosis from subthalamic nucleus deep brain stimulator lesion effect. Surg Neurol Int 4:7, 2013

19. Wooten GF, Currie LJ, Bovbjerg VE, Lee JK, Patrie J: Are men at greater risk for Parkinson's disease than women? J Neurol Neurosurg Psychiatry 75:637-639, 2004

20. Yoritaka A, Shimo Y, Takanashi M, Fukae J, Hatano T, Nakahara T, et al: Motor and non-motor symptoms of 1453 patients with Parkinson's disease: prevalence and risks. Parkinsonism Relat Disord 19:725-731, 2013

21. Zonana J, Zimmerman M, McCarty SS, Ferrando S: A case of abrupt-onset apathy, psychosis, and depression following deep brain stimulation in a patient with Parkinson's disease. Psychosomatics 52:463-467, 2011

\section{Author Contributions}

Conception and design: Sunshine, Grill, Anderson. Acquisition of data: Qureshi, Anderson, Grill. Analysis and interpretation of data: Qureshi, Cheng, Anderson, Wu, Sunshine. Drafting the article: Qureshi, Wu. Critically revising the article: Qureshi, Cheng, Wu, Pontone, Cascella, Lenz, Anderson. Reviewed submitted version of manuscript: Qureshi, Anderson. Approved the final version of the manuscript on behalf of all authors: Qureshi. Statistical analysis: Qureshi. Administrative/technical/material support: Qureshi, Cheng, Sunshine, Wu, Pontone, Lenz, Grill, Anderson. Study supervision: Sunshine, Anderson.

\section{Correspondence}

Azam A. Qureshi, University of Maryland School of Medicine, 685 W. Baltimore St., Baltimore, MD 21201. email: azam. qureshi@som.umaryland.edu. 